\title{
Meta
}

Journal des traducteurs

Translators' Journal

\section{Spécificités linguistiques de la lexicologie et de la terminologie. Nature épistémologique}

\section{Enilde Faulstich}

Volume 41, numéro 2, juin 1996

Traduction et terminologie au Brésil

Translation and Terminology in Brazil

URI : https://id.erudit.org/iderudit/002732ar

DOI : https://doi.org/10.7202/002732ar

Aller au sommaire du numéro

Éditeur(s)

Les Presses de l'Université de Montréal

ISSN

0026-0452 (imprimé)

1492-1421 (numérique)

Découvrir la revue

Citer cet article

Faulstich, E. (1996). Spécificités linguistiques de la lexicologie et de la terminologie. Nature épistémologique. Meta, 41(2), 237-246.

https://doi.org/10.7202/002732ar
Résumé de l'article

Dans cet article, nous analyserons, d'un point de vue théorique, les spécificités linguistiques de la LEXICOLOGIE et de la TERMINOLOGIE, ainsi que certains príncipes qui délimitent la nature épistémologique du LEXEME et du TERME. D'un autre côté, d'un point de vue pratique, nous commenterons quelques-unes des méthodologies utilisées au cours de recherches lexicologiques et terminologiques et ensuite nous présenterons des applications pratiques. Pour synthétiser notre pensée, nous adopterons le processus suivant : 1 . examen des principes qui régissent l'évaluation bipartite de l'unité lexicale; 2 . analyse de la genèse linguistique du lexeme et du terme ; 3. réflexion sur la nature épistémologique du lexeme et du terme ; 4. commentaires généraux sur les méthodes de travail de la lexicologie et de la terminologie ; 5. application pratique. 


\title{
SPÉCIFICITÉS LINGUISTIQUES DE LA LEXICOLOGIE ET DE LA TERMINOLOGIE. NATURE ÉPISTÉMOLOGIQUE
}

ENILDE FAULSTICH

Universidade de Brasília (LIV), Brasília, Brésil

\begin{abstract}
Résumé
Dans cet article, nous analyserons, d'un point de vue théorique, les spécificités linguistiques de la LEXICOLOGIE et de la TERMINOLOGIE, ainsi que certains principes qui délimitent la nature épistémologique du LEXÉME et du TERME. D'un autre côté, d' un point de vue pratique, nous commenterons quelques-unes des méthodologies utilisées au cours de recherches lexicologiques et terminologiques et ensuite nous présenterons des applications pratiques.

Pour synthétiser notre pensée, nous adopterons le processus suivant: 1 . examen des principes qui régissent l'évaluation bipartite de l'unité lexicale; 2 . analyse de la genèse linguistique du lexème et du terme; 3. réflexion sur la nature épistémologique du lexème et du terme; 4. commentaires généraux sur les méthodes de travail de la lexicologie et de la terminologie ; 5. application pratique.
\end{abstract}

\section{Abstract}

This article deals with the specific nature of lexicology and terminology and certain principles defining the epistemological nature of lexemes and terms. It then turns to practical concerns with a discussion of certain methodological approaches used in lexicological and terminological research, and a demonstration of practical applications.

The article is structured as follows: 1. study of the principles underlying the dual reality of lexical units; 2 . analysis of the linguistic origin of lexemes and terms; 3 . considerations on the epistemological nature of lexemes and terms; 4 . general discussion of methodological approaches in lexicology and terminology; 5 . practical application.

\section{INTRODUCTION}

Les définitions courantes du lexème et du terme sont bien connues. Un lexème est une unité lexicale du domaine du lexique général de la langue; un terme est aussi une unité lexicale, mais spécifique aux différents domaines du vocabulaire scientifique et technique. Ces éclaircissements nous permettent au départ de discerner la nature épistémologique du lexème et du terme. Examinons toutefois quels principes régissent la conception bipartite de l'unité lexicale.

\section{LA CONCEPTION BIPARTITE DE L'UNITÉ LEXICALE}

Une unité lexicale est, a priori, un signe. Selon la définition saussurienne consacrée, une langue est un système de signes et un signe se définit comme tel au sein d'un ensemble d'autres signes dégageant de ce jeu d'oppositions sa valeur et sa signification. D'après cette opinion, il est possible de reconnaître que, dans une matière donnée, lexème et terme acquièrent l'autonomie linguistique, car ils appartiennent nettement à leur 
domaine exclusif. Le lexème est l'unité de la lexicologie; le terme est l'unité de la terminologie. Cabré (1992: 75) déclare que:

la gran quantitat de característiques comunes que presenten la lexicologia i la terminologia permet de tractar-les almenys com a matèries afins [...] pero no tot són coincidències entre aquestes dues matèries, que per a alguns són part l'una de l'altra $i$ per a d'altres són matèries diferenciades. Algunes característiques divergents donen prou suport per tractarles separadament : a) el camp de treball ; b) la unitat de base ; c) els objectius aplicats ; d) el mètode de treball.

\section{LA GENÈSE DU LEXÈME ET DU TERME}

Il faut comprendre que la genèse du lexème et du terme se situe dans un contenu conceptuel, dont le premier est l'unité virtuelle qui se réalise sous la forme de «mot» du discours, et le second est «unité signifiante [...] qui désigne une notion de façon univoque à l'intérieur d'un domaine» (Boutin-Quesnel et al. 1985: 20). De cette façon nous chercherons à démontrer, à l'aide du diagramme annexé, que le concept de l'unité lexicale classifie de nouvelles unités sémantiques, ayant pour base l'insertion du lexème et du terme dans l'univers de la langue commune (langue) ou dans l'univers d'une langue propre aux spécialistes (science/technologie), respectivement.

Par conséquent, à la lumière de la pensée de Guilbert (1973:5-17), il est possible d'établir des paramètres de l'application du lexème, en tenant compte du fait que le lexème est l'unité de la langue commune.

Ainsi, les signes du lexique commun sont, d'une manière générale, porteurs de connotations psychologiques et sociales, ce qui les expose en grande partie à une multiplication de signifiés, c'est-à-dire à la polysémie. Il fait également partie de la nature de la langue commune d'attribuer des équivalences de signification à l'unité lexicale, d'où la synonymie.

De cette manière, la façon d'impliquer le lexème comme signe trouve sa spécificité dans un contexte multi-référentiel, ce qui favorise quelquefois l'ambiguîté; du point de vue fonctionnel, le lexème trace son caractère sémantique et lexicographique à partir d'une structure paradigmatique, car ce sont les oppositions distinctives qui délimitent la configuration sémantique et marquent la valeur du lexème. De cet aperçu, la lexicologie obtient une légitimité propre, bien qu'elle soit une discipline qui, tenant compte du lexique systématisé, capte de l'univers référentiel toutes les possibilités sémantiques accréditées au lexème et, de là, les conditions de celui-ci apparaissent en champs lexicaux sémasiologiques différenciés.

Poursuivant dans le même sens, faisons référence à la déclaration de Saussure, dans son ouvrage classique, où il affirme que «dans la langue chaque terme a sa valeur par son opposition avec tous les autres termes» (1972: 126) et renchérit en disant que «la langue est un système dont tous les termes sont solidaires et où la valeur de l'un ne résulte que de la présence simultanée des autres» (p. 159).

Les oppositions systématiques de niveau inférieur à celui du «mot» font également partie de la nature linguistique. Ainsi, il est naturel que, dans un schéma formateur d'unités lexicales, il existe des mécanismes qui répondent aux nécessités de nouvelles formations, au-delà des recours traditionnels calqués sur la morphologie lexicale. De nouvelles réponses proviennent des nominalisations des phrases, des recours de la valence sémantique, de la dérivation et de la composition du mot, enfin d'une structuration du lexique dans le cadre de la linguistique fonctionnelle. Suivant cet exposé, il convient de vérifier si le parcours du terme est le même que celui du lexème; enfin, un terme est-il ou non une unité lexicale? 
Boutin-Quesnel et al. (1985: 20) soulignent que «terme est aussi appelé unité terminologique». Cela dit, il est clair qu'il y a divergence entre unité lexicale et unité terminologique.

Insérée dans l'univers de la langue scientifique et technique, une unité terminologique trouve sa signification spécifique circonscrite par un des sous-codes du code majeur, c'est-à-dire qu'elle se contextualise à l'intérieur d'une langue de spécialité qui est, d'après Kocourek (1982: 31$)$, «une des sous-langues de la langue tout entière; elle a certaines ressources en commun avec la langue usuelle, mais elle a aussi ses ressources propres». définie.

Ainsi, dans cet encadrement spécifique, le terme assume le statut d'unité lexicale

Or, l'ensemble des caractéristiques jusqu'ici exposées suggère déjà que la conception épistémologique du lexème et du terme est de nature différente.

\section{NATURE ÉPISTÉMOLOGIQUE DU LEXÈME ET DU TERME}

Du point de vue de la linguistique théorique, il est possible de tracer des limites pour différencier le lexème du terme. Dans le diagramme annexé, nous pouvons démontrer de façon abrégée le cadre conceptuel représentatif de ces limites. Ainsi, à partir d'une unité abstraite unique - l'unité lexicale - nous distinguerons deux types d'éléments significatifs : le lexème et le terme.

$\mathrm{Au}$ lexème revient le statut d'unité virtuelle qui se concrétise dans l'emploi commun que les usagers font de la langue et qui, organisé systématiquement, structure les modèles lexicaux; de son côté, le terme est l'unité réelle de(s) la(les) langue(s) de spécialité, dont l'ensemble systématique forme une structure terminologique qui doit correspondre au système conceptuel d'une spécialité. Cette correspondance s'exerce par le moyen de relations taxinomiques ou formelles et/ou sémantiques entre concept, terme et rapport, motivée dans la production textuelle. De cette manière, la nature épistémologique du lexème et celle du terme se fondent justement pour atteindre ces objectifs.

La lexicologie théorique vise donc la construction d'un modèle de composantes lexiques de la grammaire prévoyant des mécanismes systématiques et adéquats pour la cohérence entre ce modèle et d'autres composantes grammaticales. Le but ultime de ce processus est la création de conditions d'uniformisation dans la formation de nouvelles unités lexicales en accord avec les principes structuraux systématiques. Une fois organisés, de tels principes évolueront comme une norme lexicale utile pour la nomination des «objets» de la langue générale.

Pour établir les objectifs de la terminologie, il convient d'observer qu'il s'agit d'une discipline d'intersection, pour laquelle on trouve peu d'interprétations scientifiques de valeur. Toutefois, observée du point de vue de la linguistique, ce qui effectivement nous intéresse, la terminologie possède dans le langage sa science de base. Observée du point de vue d'une discipline scientifico-technique, la terminologie est le réflexe formel de son organisation conceptuelle (Cabré 1992: 73). Dans cette perspective, le langage terminologique a pour objectif la dénomination des «objets» créés dans l'univers qui se sert de la «langue scientifique» ou langue de spécialité. Rappelons que, pour créer un terme, l'inventeur se sert de la lexicologie, de la morphologie, de la sémantique enfin, des composantes lexiques et grammaticales. Par conséquent, «terminologia és llenguatge» comme l'affirme Cabré (1992: 72). 


\section{COMMENTAIRES GÉNÉRAUX SUR LES MÉTHODES DE TRAVAIL DE LA LEXICOLOGIE ET DE LA TERMINOLOGIE}

Cet exposé nous permet de dire que les principes délimitant le lexème et le terme proviennent davantage des recours méthodologiques de la lexicologie et de la terminologie que des fondements conceptuels du lexème et du terme.

La méthode de travail de la lexicologie tient compte des hypothèses théoriques qui réfutent ou acceptent les productions des usagers au moyen d'échantillonnages; la terminologie ancienne n'expliquait pas le comportement linguistique des usagers; cependant la «nouvelle terminologie» - la socioterminologie - a pour but d'étudier le terme dans une perspective linguistique, vers l'interaction sociale.

Ainsi, les méthodes de la lexicologie théorique et celles de la lexicologie descriptive servent de point de départ pour systématiser de diverses manières les vocabulaires, tout comme elles servent de base au travail lexicographique dans l'élaboration de dictionnaires de la langue générale. Voilà pour les résultats pratiques! Par contre, les méthodes de la terminologie servent de point de départ pour la terminographie, laquelle a pour but la constitution de dictionnaires spécialisés.

\section{APPLICATIONS PRATIQUES DE LA LEXICOLOGIE ET DE LA TERMINOLOGIE}

Au cours des années passées, nous avons développé des recherches fondées sur le modèle lexicologique et terminologique.

\section{LE MODÈLE LEXICOLOGIQUE}

Une expérience intéressante est celle qui nous permet de conjuguer concepts et méthodes sur les champs - sémantiques et lexicaux - avec une proposition de systématisation de vocabulaire tiré d'un corpus organisé en champs lexicaux.

Le corpus a été constitué à partir de la presse brésilienne qui traite de faits divers : criminalité, armes, police, etc. Nous avions pour but de vérifier si les concepts de lexie et de lexème pouvaient remplir leur fonction dans un cadre conceptuel typique.

La recherche se divise en quatre parties: 1) au début, nous examinons les concepts de lexie (Pottier) et de lexème (Coseriu); 2) ensuite, nous mettons en discussion la théorie des champs lexicaux (Trier-Weisgerber, Porzig, Coseriu, Pottier, Lyons, Fillmore) en relevant toutes les difficultés théoriques et pratiques que demandent les différentes organisations structurales; 3) par après, nous organisons le corpus récolté dans les diagrammes dans une tentative d'établissement des modèles pour l'organisation du vocabulaire systématique; 4 ) enfin, nous transcrivons toutes les phrases: soit 874 contextes où les lexies sont insérées.

Un des aspects importants de la méthodologie employée est que le sens de chaque lexie qui compose le corpus a été comparé avec les signifiés courants dans les dictionnaires Novo Dicionário Aurélio da Língua Portuguesa (1975) et Novo Dicionário Brasileiro Melhoramentos (1969), afin de rendre conforme l'usage des lexies dans le discours des quotidiens ou d'enregistrer un nouveau signifié qui provienne de ce contexte.

Les lexies étudiées issues du «langage des avis de police» sont structurées en champs lexicaux, que nous transcrirons en portugais (Faulstich 1980):

a. Macrocampo - I. Infrator

I.l. (Campo de) Criminoso; I.2. Autoria do crime; 1.3. Grupo de infratores; I.4. Preso; I.5. Rixa; 1.6. Cena de violência; 1.7. Transar; I.8. Agredir.

b. Macrocampo-II. Infração / Ocorrência

II.1. Negócio nefando; II.2. Crime; II.3. Homicídio; II.4. Vias de fato; II.5. Droga; II.6. Ocorrência. 
c. Macrocampo-III. Policia

III.I. Autoridade; III.2. Grupamento; III.3. Operação; III.4. Receber denúncia; III.S. Capturar ; III.6. Vigilância.

d. Macrocampo - IV. Vítima

IV.1. Ficar ferida; IV.2. Cadáver; IV.3. Legítima defesa.

e. Macrocampo - V. Arma

V.1. Arma de fogo; V.2. Arma branca; V.3. Arma (contundente); V.4. Bomba.

f. Macrocampo - VI. Local

VI.I. Polícia; VI.2. Infrator; VI.3. Vitima; V.4. Crime.

g. Macrocampo-VII. MultidãoVII.1. Multidão

h. Macrocampo - VIII. Justiça

VIII.1. Acusação; VIII.2. Juizo; VIII.3. Crime.

Ensuite, nous exposerons un modèle de champ lexical - le I.8. Agredir — pour démontrer la structuration que nous ont donnée les lexies récoltées :

Ao Agredir, o agressor, em relação à vítima, pode 1. cobrir ; 2 . investir ; 3. imobilizar: 4. executar.

Para cobrir, é preciso andar coberto.

Para investir, é preciso dar o bote, ou cuspir fogo e, ao cuspir fogo, pode o agressor ou atirar a esmo, ou atirar a torto e a direito, ou travar cerrada fuzilaria. algemar.

Para imobilizar, é preciso manietar ou amordaçar e, ao manietar, é preciso

Para executar, é preciso ou abater, ou esfaquear, ou estrangular. Ao abater, pode o agressor crivar (de balas), ou chacinar. Todas estas açóes convergem para uma única direção, que é desfigurar a vítima.

\section{LE MODÈLE TERMINOLOGIQUE}

Il n'y a pas, au Brésil, de tradition en recherche terminologique. Mais il existe maintenant dans les universités des groupes qui ont mis en marche des travaux de cette nature.

À l'Université de Brasília, je suis responsable d'une «Ligne de Recherche» qui s'appelle Léxico e Terminologia. Il s'agit d'un programme de maîtrise en linguistique.

Notre but est de développer une recherche pour la conception d'un dictionnaire spécialisé, à partir des méthodes normales de travail terminologique et terminographique. Le corpus est constitué de termes pris dans des textes scientifiques et techniques du domaine de la génétique, plus précisément l'amélioration génétique des plantes.

Nous avons rédigé une méthodologie appropriée pour l'élaboration du glossaire, n'ayant que trop tardivement eu connaissance de l'existence de telles méthodologies produites à l'Office de la langue française du Québec et à l'Université Laval. De plus, nous avons créé une fiche terminologique adéquate qui nous permet d'enregistrer les données spécifiques du terme pertinent à un domaine scientifique ou technique. Nous avons aussi rédigé un document où nous exposons les termes en système de renvois.

Pour illustrer notre démarche, voici un exemple de renvoi extrait du glossaire en cours d'élaboration (Faulstich 1992). La formulation est en portugais.

Ordenação sistemática de termos

A ordenação dos termos de maneira sistemática resulta da classificação desses termos de acordo com a noção semântica chave que os agrupa (TERMCAT 1990: 66). Um dos requisitos fundamentais para essa ordenação é a rede de remissivas que organiza naturalmente 
os campos léxicos. As vantagens desse tipo de estrutura são várias, entre outras, consideramos que é possível (i) haver ao largo do trabalho melhor controle da pertinência dos termos dentro de cada campo; (ii) haver maior coerência e maior rigor na definição de cada um; (iii) consultar imediatamente os termos relacionados e (iv) organizar famîlias terminológicas que têm traços onomasiológicos comuns, cujos termos se aproximam por inclusão ou por oposição. Desse modo, a ordenação de cada rede forma microcampos dentro de um macrocampo.

A título de exemplo, apresentaremos a rede de remissivas que parte de DIVISÃo CELULAR, redigida em ordenação sistemática, em que $V$, significa VER/CONSULTAR A REMISSIVA. Cada verbete segue o paradigma: termo-entrada $=+$ categoria gramatical + gênero + definição + fonte _ contexto _ fonte _ remissiva(s).

divisão celular. S.f. Modo de reprodução das células por divisão direta ou amitose e por divisão indireta ou mitose. F. \& A. Na divisão celular, a célula reparte todo seu conteúdo por duas células-filhas, iguais entre si. Dic. Téc. Melh., 47. V. amitose; mitose.

amitose. S.f. Divisão celular direta e simples, por estrangulamento do núcleo da célula. F. \& A. A amitose é própria de células envelhecidas. Dic. Téc. Melh., 7. V. divisão celular; mitose

mitose. S.f. Divisão celular indireta em que o núcleo forma cromossomos que sc partem pelos processos de prófase, metáfase, anáfase e telófase. Adap. Novo Dic. Aurélio. Uma mitose dura entre $30 \mathrm{~m}$ e $3 \mathrm{~h}$. Dic. Téc. Melh., 110. V. divisão celular; cromossomo ; prófase ; metáfase ; anáfase ; telófase ; cariocinese ; amitose

cromossomo. S.m. Estrutura nucleoprotéica observada durante as divisões celulares. Gen. Agropec., 344. O cromossomo possui um filamento enrolado em espiral e uma matriz, massa protetora. Dic. Téc. Melh., 39. V. divisão celular

prófase. S.f. Primeira fase da mitose donde os cromossomos se individualizam em filamentos longitudinais. Gen. Agr., 351. A prófase caracteriza-se, entre outros fatos, pela condensaçấo dos cromossomos. Op. cit. idem. V. mitose; metáfase; anáfase ; telófase ; cromossomo

metáfase. S.f. Segunda fase da mitose donde os cromossomos alinham-se em posição equatorial da célula, mas mantêm-se presos às fibras do fuso. $\mathbf{F}$. \& $\mathbf{A}$. A metáfase se realiza na placa equatorial do fuso acromático. Dic. Téc. Melh., 110. V. mitose; fuso; prófase ; anáfase ; telófase ; cromossomo

fuso. S.m. Feixe de fibrilas citoplasmáticas, visíveis na célula durante a mitose. Dic. Téc. Melh., 73. V. metáfase ; anáfase

anáfase. S.f. Terceira fase da mitose em que ocorre a segregação dos cromossomos para os pólos opostos da célula. F. \& A. Na anáfase, os cromossomos-filhos afastam-se em direção aos pólos do fuso. Dic. Téc. Melh. 110. V. mitose; prófase; metáfase; telófase; cromossomo

telófase. S.f. Quarta fase da mitose donde se constituem os núcleos das células-filhas e se forma uma nova membrana. F. \& A. Na telófase, as duas células-filhas possuem núcleos interfásicos. Le Petit Larousse, 1993, p. 996. V. mitose; prófase ; metáfase ; anáfase ; cromossomo

cariocinese. S.f. Divisão do núcleo da célula. F. \& A. V. mitose

\section{CONCLUSION}

En conclusion, nous pouvons affirmer que :

1. l'ensemble des lexèmes conduit à l'organisation de ceux-ci en champs sémasiologiques; déjà l'ensemble de termes peut former des champs onomasiologiques, justement parce qu'ils s'insèrent dans l'univers de la langue scientifique et technique;

2. la distinction épistémologique du lexème et du terme provient davantage des recours méthodologiques que des fondements conceptuels de chacun;

3. la nature épistémologique du lexème et du terme se confirme dans le développement des travaux pratiques qui utilisent les méthodes appropriées. 


\section{RÉFÉRENCES}

Actas del Primer Simposio Latino-americano de Terminología (1988): Grupo de Investigación Terminológica (GIT), Caracas, Universidad Simón Bolívar.

AUGER, P., ROUSSEAU, L. et al. (1978): Méthodologie de la recherche terminologique, Québec, Éditeur officiel du Québec.

BOULANGER, J.-C. (1982) : «Synonymie, néonymie et normalisation en terminologie», Problèmes de la définition et de la synonymie en terminologie, Actes du Colloque International de Terminologie, Québec, Université Laval, TERMIA, pp. 15-34.

BOUTIN-QUESNEL, R. et al. (1985): Vocabulaire systématique de la terminologie, Québec, Publications du Québec.

CABRÉ, M. T. (1992) : La terminologia. La teoria, els mètods, les aplicacions, Barcelona, Empúries.

CÉLESTIN, T., GODBOUT, G. et P. VACHON-L'HEUREUX (1984): Méthodologie de la recherche terminologique ponctuelle, Québec, Office de la langue française.

CORBEIL, J.-C. (1982): «Définition et synonymie en terminologie», Problèmes de la définition et de la synonymie en terminologie, Actes du Colloque Intemational de Terminologie, Université Laval (Québec), TERMIA, pp. 3-11.

DROZD, M. L. (1982) : «Terminological Definitions : Characteristics and Demands», Problèmes de la définition et de la synonymie en terminologie, Actes du Colloque International de Terminologie, Université Laval (Québec), TERMIA, pp. 37-100.

DUBUC, R. (1982) : «Synonymie et terminologie», Problèmes de la définition et de la synonymie en terminologie, Actes du Colloque International de Terminologie, Université Laval (Québec), TERMIA, pp. $193-206$.

FAULSTICH, E. (1980): Lexicologia : a linguagem do noticiário policial, Brasília, Horizonte.

FAULSTICH, E. (1991): «Apreensão de conceitos neológicos / semânticos», Reflexão - Campus, Brasília, FAC/UnB.

FAULSTICH, E. (1992): «Elaboração de glossário de termos de Melhoramento Genético de Plantas», Anais do III Simpósio Ibero-americano de Terminologia, RITerm '92, San Millán de la Cogolla, Espanha.

FAULSTICH, E. (1992) : «Metodologia para projeto terminográfico», Anais do II Simpósio Latino-Americano de Terminologia e I Encontro Brasileiro de Terminologia Técnico-Científica, Brasilia, IBICT, Paris, Union Latine, pp. 425-432.

FAULSTICH, E. (1992): «Rede de remissivas em um glossário técnico», Anais do VI Encontro Nacional da ANPOLL, Porto Alegre.

FAULSTICH, E. (1993): «Natureza epistemológica do lexema e do termo», XLI Anais de Seminário do GEL, São Paulo.

GOUADEC, D. (1990): Terminologie, Constitution de données, Paris, Afnor.

GUILBERT, L. (1973): «La spécificité du terme scientifique et technique», Les vocabulaires techniques et scientifiques, Langue française, 17, pp. 5-17.

KOCOUREK, R. (1982): La langue française de la technique et de la science, Wiesbaden, Brandstetter.

LEHMANN, A. (1990): «De définition à définition. L'interprétation dans le dictionnaire par le jeu des renvois», La définition, Paris.

REY, A. (1979): La terminologie nom et notions, Paris, PUF.

RONDEAU, G. et H. FELBER (dir.) (1981): Textes choisis de terminologie, Québec, GIRSTERM.

ROUSSEAU, L..J. (1982): «La définition terminologique», Problèmes de la définition et de la synonymie en terminologie, Actes du Colloque International de Terminologie, Université Laval (Québec), TERMIA, pp. 35-46.

SAGER, J. C. (1982): «Definitions in Terminology», Problèmes de la définition et de la synonymie en terminologie, Actes du Colloque International de Terminologie, Université Laval (Québec), TERMIA, pp. 113-139.

SAUSSURE, F. (1972): Cours de linguistique générale, Paris, Payot.

TERMCAT, Centre de terminologia (1990): Metodologia del treball terminologic, Catalunya, Departamento de Cultura. 
DIAGRAMME 1

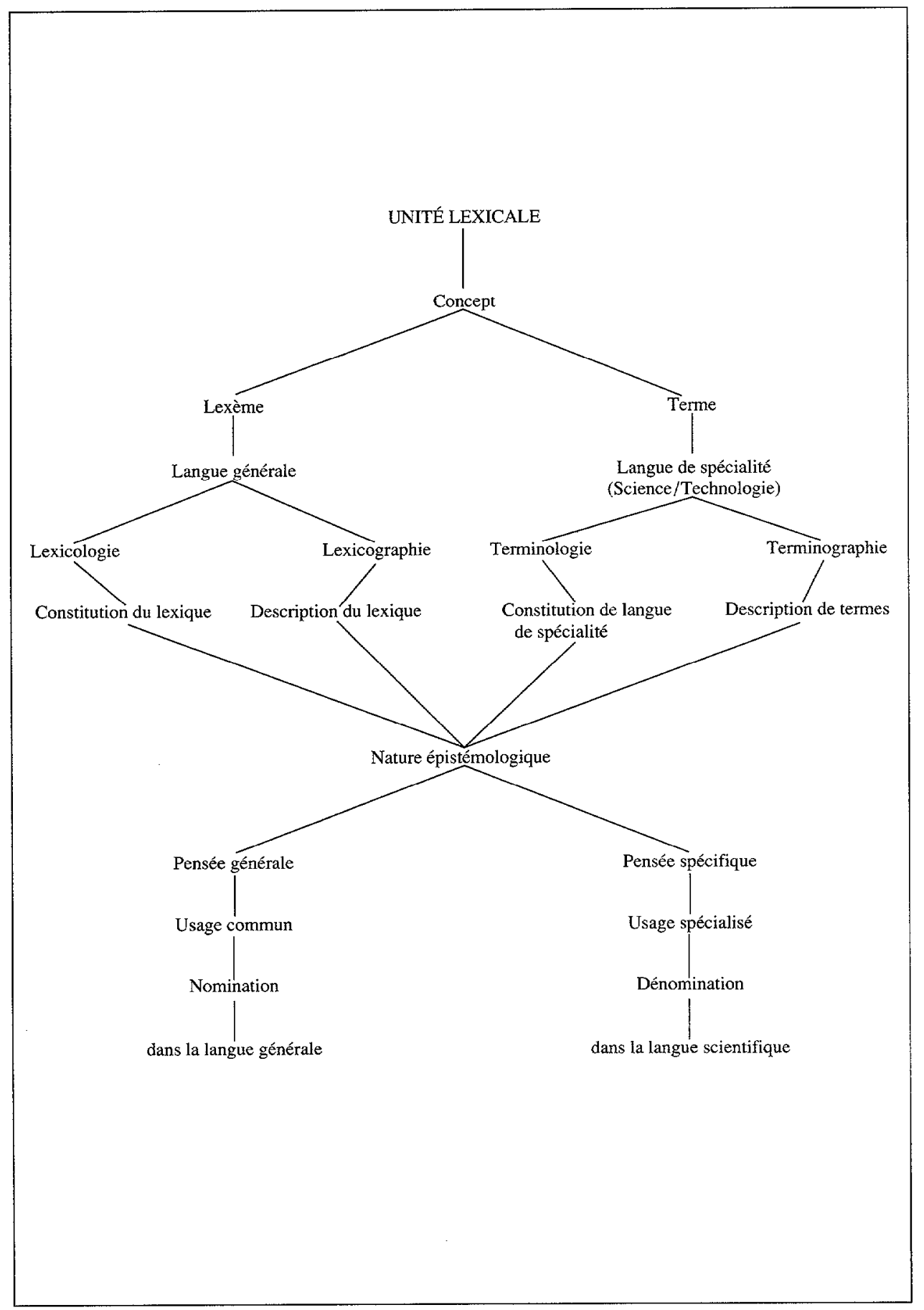


DIAGRAMME 2

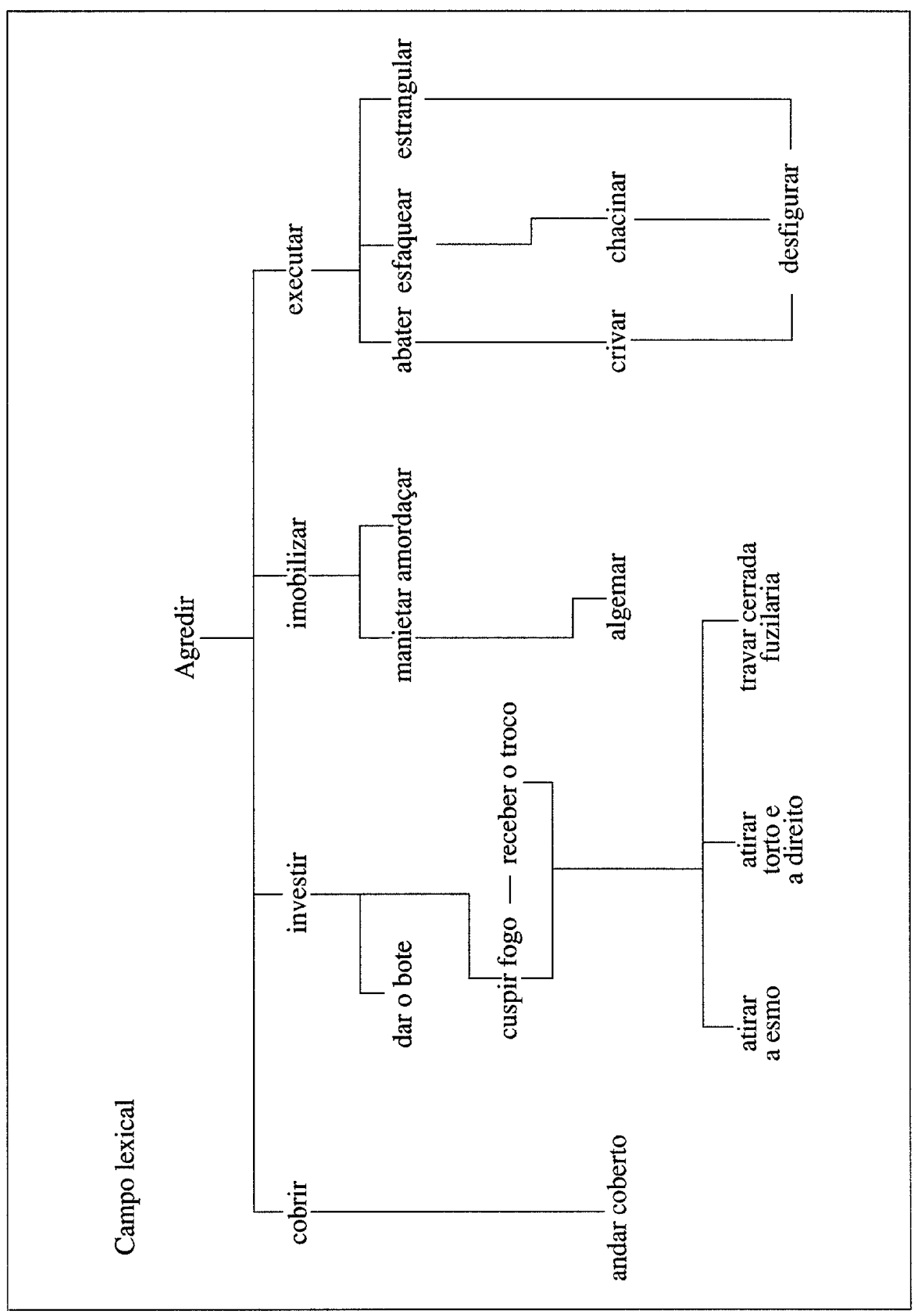


DIAGRAMME 3

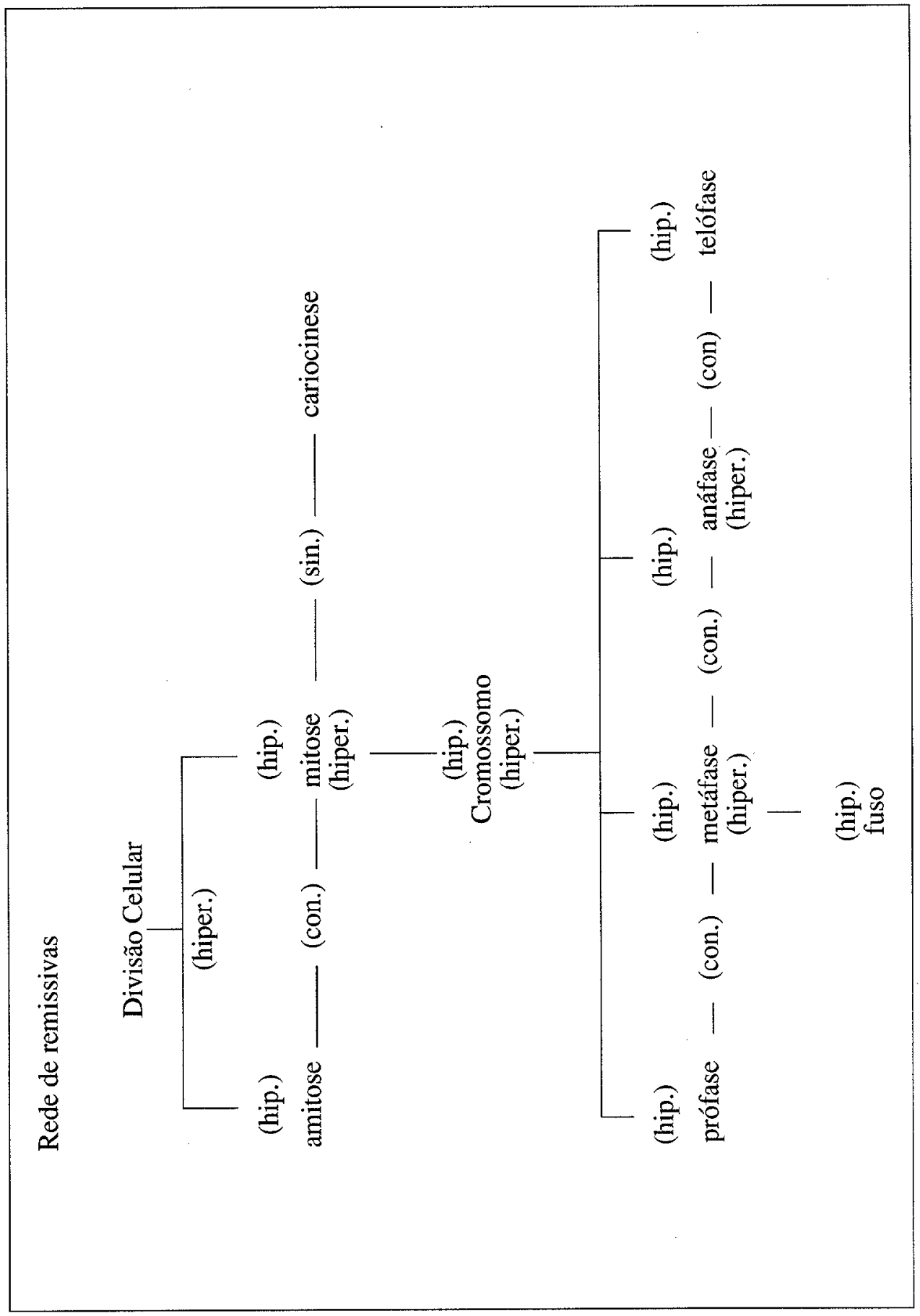

